\title{
A Partially Synchronizing Coloring ${ }^{\star}$
}

\author{
A.N. Trahtman ${ }^{\star \star}$ \\ Bar-Ilan University, Dep. of Math., 52900, Ramat Gan, Israel \\ Lecture Notes in Computer Science, 6072(2010), 363-370
}

\begin{abstract}
Given a finite directed graph, a coloring of its edges turns the graph into a finite-state automaton. A $k$-synchronizing word of a deterministic automaton is a word in the alphabet of colors at its edges that maps the state set of the automaton at least on $k$-element subset. A coloring of edges of a directed strongly connected finite graph of a uniform outdegree (constant outdegree of any vertex) is $k$-synchronizing if the coloring turns the graph into a deterministic finite automaton possessing a $k$-synchronizing word.

For $k=1$ one has the well known road coloring problem. The recent positive solution of the road coloring problem implies an elegant generalization considered first by Béal and Perrin: a directed finite strongly connected graph of uniform outdegree is $k$-synchronizing iff the greatest common divisor of lengths of all its cycles is $k$.

Some consequences for coloring of an arbitrary finite digraph are presented. We describe a subquadratic algorithm of the road coloring for the $k$-synchronization implemented in the package TESTAS. A new linear visualization program demonstrates the obtained coloring. Some consequences for coloring of an arbitrary finite digraph and of such a graph of uniform outdegree are presented.
\end{abstract}

Keywords: graph, algorithm, synchronization, road coloring, deterministic finite automaton.

\section{Introduction}

The famous road coloring problem was stated almost 40 years ago [1] for a strongly connected directed graph of uniform outdegree where the greatest common divisor ( $\mathrm{gcd}$ ) of lengths of all its cycles is one.

Together with the Cerny conjecture [7], [9], the road coloring problem was once one of the most fascinating problems in the theory of finite automata. In the popular Internet Encyclopedia "Wikipedia" it is on the list of most interesting unsolved problems in mathematics. The recent positive solution of the road coloring conjecture [10], [13], [11] has posed a lot of generalizations and new problems.

* http://www.cs.biu.ac.il/ trakht

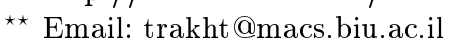


One of them is $k$-synchronizing coloring. A solution of the problem based on the method from [11] was appeared first in [4] and repeated later independently in $[5],[12]$.

Some consequences for coloring of an arbitrary finite digraph as well as for coloring of such a graph of a uniform outdegree are a matter of our interest. The minimal value of $k$ for $k$-synchronizing coloring exists for any finite digraph and therefore a partially synchronizing coloring can be obtained.

Here we describe a polynomial time algorithm for $k$-synchronizing coloring. Our proof also is based on [11] and [12], the more so the proofs in [4] meanwhile have some gaps. The theorems and lemmas from [11] are presented below without proof. The proofs are given only for new or modified results. The realization of the algorithm is demonstrated by a new linear visualization program [3]. For an $n$-state digraph with uniform outdegree $d$, the time complexity of the algorithm is $O\left(n^{3} d\right)$ in the worst case and $O\left(n^{2} d\right)$ in the majority of cases. The space complexity is quadratic.

\section{Preliminaries}

As usual, we regard a directed graph with colors assigned to its edges as a finite automaton, whose input alphabet $\Sigma$ consists of these colors.

A directed graph with constant outdegree (the number of outgoing edges) of all its vertices is called a graph of uniform outdegree.

A finite directed strongly connected graph of uniform outdegree where the gcd of lengths of all its cycles is $k$ will be called $k$-periodic.

An automaton is deterministic if no state has two outgoing edges of the same color. In a complete automaton each state has outgoing edges of any color.

If there exists a path in an automaton from the state $\mathbf{p}$ to the state $\mathbf{q}$ and the edges of the path are consecutively labelled by $\sigma_{1}, \ldots, \sigma_{k} \in \Sigma$, then for $s=\sigma_{1} \ldots \sigma_{k} \in \Sigma^{+}$let us write $\mathbf{q}=\mathbf{p} s$.

Let $P s$ be the map of the subset $P$ of states of an automaton using the word $s \in \Sigma^{+}$. For the transition graph $\Gamma$ of an automaton let $\Gamma s$ denote the map of the set of states of the automaton.

Let $|P|$ denote the size of the subset $P$ of states from an automaton (of vertices from a graph).

A word $s \in \Sigma^{+}$is called a $k$-synchronizing word of the automaton with transition graph $\Gamma$ if both $|\Gamma s|=k$ and for all words $t \in \Sigma^{*}$ holds $|\Gamma t| \geq k$.

A coloring of a directed finite graph is $k$-synchronizing if the coloring turns the graph into a deterministic finite automaton possessing a $k$-synchronizing word and the value of $k$ is minimal.

A pair of distinct states $\mathbf{p}, \mathbf{q}$ of an automaton (of vertices of the transition graph) will be called synchronizing if $\mathbf{p} s=\mathbf{q} s$ for some $s \in \Sigma^{+}$. In the opposite case, if for any $s \mathbf{p} s \neq \mathbf{q} s$, we call the pair deadlock.

A synchronizing pair of states $\mathbf{p}, \mathbf{q}$ of an automaton is called stable if for any word $u$ the pair of states $\mathbf{p} u, \mathbf{q} u$ is also synchronizing [6], [8]. 
We call the set of all outgoing edges of a vertex a bunch if all these edges are incoming edges of only one vertex.

The subset of states (of vertices of the transition graph $\Gamma$ ) of maximal size such that every pair of states from the set is a deadlock will be called an F-clique.

\section{A $k$-synchronizing coloring}

Lemma 1. Let a finite directed strongly connected graph $\Gamma$ with uniform outdegree have a k-synchronizing coloring. Then the greatest common divisor $d$ of lengths of all its cycles is not greater than $k$.

If the length of a path from the state $\mathbf{p}$ to the state $\mathbf{q}$ is equal to $i \neq 0$ (modulo d) then for any word $s$ one has $\mathbf{p} s \neq \mathbf{q} s$.

Proof. Let $N$ be the function defined on the states of $\Gamma$ in the following way we take an arbitrary vertex $\mathbf{p}$ and let $N(\mathbf{p})=0$. Then for each vertex $\mathbf{q}$ with defined $N(\mathbf{q})$ suppose for any next nearest vertex $\mathbf{r} N(\mathbf{r})=N(\mathbf{q})+1$ (modulo $d)$. The graph is strongly connected, whence for each vertex the function $N$ is defined. The enumeration does not imply a contradiction anywhere because the length of each cycle is divided by $d$. Then by any coloring the mapping by a word $t$ produced the same shift of size $|t|$ (modulo $d$ ) of the function $N$ on the states. Therefore the states with distinct values of $N$ could not have common image and $|\Gamma t| \geq d$ for any word $t .|\Gamma s|=k$ for $k$-synchronizing word $s$. Consequently, $k \geq d$.

By any coloring, the mapping by a word $s$ produced the same shift of the function $N$ on the set of states. $N(\mathbf{p} s)=N(\mathbf{p})+|s|$ (modulo $d$ ). Therefore the difference of the values of the function $N$ on two states is not changed by the shift.

Theorem 1. [6], [8], [10] Let us consider an arbitrary coloring of a strongly connected graph $\Gamma$ with constant outdegree. Stability of states is a binary relation on the set of states of the obtained automaton. Denote the reflexive closure of this relation by $\rho$. Then $\rho$ is a congruence relation, $\Gamma / \rho$ presents a directed strongly connected graph with constant outdegree, the gcd d of all its cycles is the same as in $\Gamma$, and a $k$-synchronizing coloring of $\Gamma / \rho$ implies a $k$-synchronizing recoloring of $\Gamma$.

Lemma 2. [10] Let $F$ be an F-clique via some coloring of a strongly connected graph $\Gamma$. For any word $s$ the set $F s$ is also an F-clique and each state [vertex] p belongs to some F-clique.

Lemma 3. Let $A$ and $B(|A|>1)$ be distinct $F$-cliques via some coloring without stable pairs of the k-periodic graph $\Gamma$. Then $|A|-|A \cap B|=|B|-|A \cap B|>1$.

Proof. Let us assume the contrary: $|A|-|A \cap B|=1$. By the definition of $F$-clique, $|A|=|B|$ and so $|B|-|A \cap B|=1$, too. Thus $|A|-|A \cap B|=|B|-|A \cap B|=1$.

The pair of states $\mathbf{p} \in A \backslash B$ and $\mathbf{q} \in B \backslash A$ is not stable. Therefore for some word $s$ the pair $(\mathbf{p} s, \mathbf{q} s)$ is a deadlock. Any pair of states from the $F$-clique $A$ 
and from the $F$-clique $B$ as well as from $F$-cliques $A s$ and $B s$ is a deadlock. So each pair of states from the set $(A \cup B) s$ is a deadlock, whence $(A \cup B) s$ is an $F$-clique.

One has $|(A \cup B) s|=|A|+1>|A|$ in spite of maximality of the size of $F$-clique $A$.

Lemma 4. [10] Let some vertex of a directed complete graph $\Gamma$ have two incoming bunches. Then any coloring of $\Gamma$ has a stable pair.

Proof. If a vertex $\mathbf{p}$ has two incoming bunches from vertices $\mathbf{q}$ and $\mathbf{r}$, then the pair $\mathbf{q}, \mathbf{r}$ is stable for any coloring because $\mathbf{q} \alpha=\mathbf{r} \alpha=\mathbf{p}$ for any letter (color) $\alpha \in \Sigma$.

\subsection{The spanning subgraph with maximum of edges in the cycles}

Definition 1. Let us call a subgraph $S$ of the k-periodic graph $\Gamma$ a spanning subgraph of $\Gamma$ if all vertices of $\Gamma$ belong to $S$ and exactly one outgoing edge of every vertex (in the usual graph-theoretic terms it is 1-outregular spanning subgraph).

A maximal subtree of the spanning subgraph $S$ with root on a cycle from $S$ and having no common edges with cycles from $S$ is called a tree of $S$.

The length of the path from a vertex $\mathbf{p}$ through the edges of its tree of the spanning set $S$ to the root of the tree is called the level of $\mathbf{p}$ in $S$.

A tree with vertex of maximal level is called a maximal tree.

Remark 1 Any spanning subgraph $S$ consists of disjoint cycles and trees with roots on cycles. Each tree and cycle of $S$ is defined identically. The level of the vertices belonging to some cycle is zero. The vertices of the trees except the roost have a positive level. The vertices of maximal positive level have no incoming edge in $S$. The edges labelled by a given color defined by any coloring form a spanning subgraph. Conversely, for each spanning subgraph, there exists a coloring and a color such that the set of edges labelled with this color corresponds to this spanning subgraph.

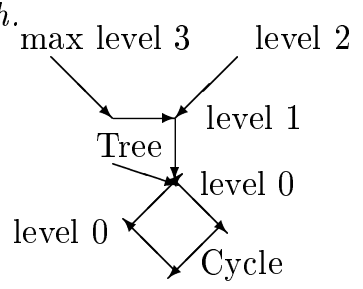

Lemma 5. Let $N$ be a set of vertices of level $n$ from some tree of the spanning subgraph $S$ of the k-periodic graph $\Gamma$. Then via a coloring of $\Gamma$ such that all edges of $S$ have the same color $\alpha$, for each $F$-clique $F$ holds $|F \cap N| \leq 1$.

Proof. Some power of $\alpha$ synchronizes all states of a given level of the tree and maps them into the root. Each pair of states from an $F$-clique could not be synchronized and therefore could not belong to $N$. 
Lemma 6. [10] Let d-periodic graph $\Gamma$ have a spanning subgraph $R$ consisting solely of disjoint cycles (without trees). Then $\Gamma$ either is a cycle of length $d$ of bunches or has another spanning subgraph with exactly one maximal tree.

Lemma 7. [11] Assume that no vertex of the graph $\Gamma$ has two incoming bunches. Let $R$ be a spanning subgraph with non-trivial tree and let its tree $T$ with the root $\mathbf{r}$ on cycle $H$ have all vertices of maximal level $L$ and one of them is the vertex p. Let us consider

1) replacing an edge e from $R$ with an edge having the same start vertex as e and ending in $\mathbf{p}$,

2) replacing in $R$ an incoming edge of a root on the path in the tree from $\mathbf{p}$,

3) replacing in $R$ an incoming edge of a root from $H$.

Suppose that at most two such operations do not increase the number of edges in cycles. Then by the coloring of $R$ by the color $\alpha \Gamma$ has a spanning subgraph with one maximal tree and the pair $\mathbf{p} \alpha^{L-1}, \mathbf{p} \alpha^{L+|H|-1}$ is stable.

Theorem 2. Any k-periodic graph $\Gamma$ of size greater than $k$ has a coloring with stable pair.

Proof. We have $|\Gamma|>k$. By Lemma 4, in the case of vertex with two incoming bunches $\Gamma$ has a coloring with stable pairs. In opposite case, by Lemmas 7 and 6, $\Gamma$ has a spanning subgraph $R$ with one maximal tree in $R$.

Let us give to the edges of $R$ the color $\alpha$ and denote by $C$ the set of all vertices from the cycles of $R$. Then let us color the remaining edges of $\Gamma$ by other colors arbitrarily.

By Lemma 2, in a strongly connected graph $\Gamma$ for every word $s$ and $F$-clique $F$ of size $|F|>1$, the set $F s$ also is an $F$-clique of the same size and for any state $\mathbf{p}$ there exists an $F$-clique $F$ such that $\mathbf{p} \in F$.

In particular, some $F$ has non-empty intersection with the set $N$ of vertices of maximal level $L$. The set $N$ belongs to one tree, whence by Lemma 5 this intersection has only one vertex. The word $\alpha^{L-1}$ maps $F$ on an $F$-clique $F_{1}$ of size $|F|$. One has $\left|F_{1} \backslash C\right|=1$ because the sequence of edges of the color $\alpha$ from any tree of $R$ leads to the root of the tree, and the root belongs to a cycle colored by $\alpha$ from $C$ and only for the set $N$ with vertices of maximal level holds $N \alpha^{L-1} \not \subset C$. So $\left|N \alpha^{L-1} \cap F_{1}\right|=\left|F_{1} \backslash C\right|=1$ and $\left|C \cap F_{1}\right|=\left|F_{1}\right|-1$.

Let the integer $m$ be a common multiple of the lengths of all considered cycles from $C$ colored by $\alpha$. So for any $\mathbf{p}$ from $C$ as well as from $F_{1} \cap C$ holds $\mathbf{p} \alpha^{m}=\mathbf{p}$. Therefore for an $F$-clique $F_{2}=F_{1} \alpha^{m}$ holds $F_{2} \subseteq C$ and $C \cap F_{1}=F_{1} \cap F_{2}$.

Thus two $F$-cliques $F_{1}$ and $F_{2}$ of size $\left|F_{1}\right|>1$ have $\left|F_{1}\right|-1$ common vertices. So $\left|F_{1} \backslash\left(F_{1} \cap F_{2}\right)\right|=1$. Consequently, in view of Lemma 3 , there exists a stable pair in the considered coloring.

Theorem 3. Every strongly connected graph $\Gamma$ is $k$-periodic if and only if the graph has a k-synchronizing coloring. For arbitrary coloring $\Gamma$ is $m$-synchronizing for $m \geq k$.

Proof. From the $k$-synchronizing coloring of $\Gamma$ by Lemma 1 follows that if $\Gamma$ is $m$-periodic graph then $m \geq k$. So $|\Gamma| \geq k$. If $|\Gamma|=k$ then also $m=k$. 
Thus we only need to consider the case $|\Gamma|>k$. By Lemmas 6 and 7 there exists a spanning subgraph with one maximal tree, whence by Theorem 2 there exists a stable pair of states. Theorem 1 reduced the problem to a homomorphic image of $\Gamma$ of smaller size and the induction finishes the proof.

The theorem 3 implies some consequences, in particular, even for an arbitrary digraph.

Lemma 8. Let a finite directed graph $\Gamma$ have a sink component $\Gamma_{1}$. Suppose that by removing some edges of $\Gamma_{1}$ one obtains strongly connected directed graph $\Gamma_{2}$ of uniform outdegree. Let $k$ be the greatest common divisor of lengths of the cycles of $\Gamma_{2}$. Then $\Gamma$ has k-synchronizing coloring.

Proof. $\Gamma_{2}$ has a $k$-synchronizing coloring by Theorem 3 . The edges outside $\Gamma_{2}$ can be colored arbitrarily, sometimes by adding new colors for to obtain deterministic automaton.

Lemma 9. Let $\Gamma_{1}, \ldots, \Gamma_{m}$ be strongly connected components of a finite directed graph $\Gamma$ of uniform outdegree such that no edge leaves the component $\Gamma_{i}(0<$ $i \leq m)$. Let $k_{i}$ be the greatest common divisor of lengths of cycles in $\Gamma_{i}$ and suppose $k=\sum_{j=1}^{m} k_{j}$. Then $\Gamma$ has k-synchronizing coloring.

The Lemmas 8, 9 imply

Theorem 4. Let finite directed graph $\Gamma$ have a subgraph of uniform outdegree with strongly connected components $\Gamma_{1}, \ldots, \Gamma_{m}$ such that no edge leaves the component $\Gamma_{i}(0<i \leq m)$ in the subgraph. Let $k_{i}$ be the greatest common divisor of lengths of cycles in $\Gamma_{i}$ and suppose $k=\sum_{j=1}^{m} k_{j}$. Then $\Gamma$ has a $k$-synchronizing coloring.

The proof for arbitrary $k$ did not use anywhere that $k \neq 1$, whence from the validity of the Road Coloring Conjecture we have the following:

Theorem 5. [11] [10] Every finite strongly connected graph $\Gamma$ of uniform outdegree with greatest common divisor of all its cycles equal to one has synchronizing coloring.

\section{$2 \quad$ Find minimal $k$ for $k$-synchronizing coloring}

The algorithm is based on Theorem 3 and Lemma 8. One must check the existence of strongly connected components $(S C C)$ having no outgoing edges to others $S C C$ and check the condition on gcd in any such $S C C H$. There exists a subgraph $S$ of $C$ of maximal uniform outdegree. Then we check the condition on gcd in any such $S$. Let us use the linear algorithm of finding strongly connected component $S C C$ [2]. Then we mark all $S C C$ having outgoing edges to others $S C C$ and study only all remaining $S C C H$.

The coloring of $H$ : We find in every $H$ the value of $k_{H}$ for the $k_{H}$-synchronizing coloring of $H$. Let $\mathbf{p}$ be a vertex from $H$. Suppose $d(\mathbf{p})=1$. For an edge $\mathbf{r} \rightarrow \mathbf{q}$ 
where $d(\mathbf{r})$ is already defined and $d(\mathbf{q})$ is not defined suppose $d(\mathbf{q})=d(\mathbf{r})+1$. If $d(\mathbf{q})$ is defined let us add the difference $a b s(d(\mathbf{q})-1-d(\mathbf{r}))$ to the set $D$ and count the gcd of the integers from $D$. If finally $g c d=k_{H}$, then the $S C C H$ has $k_{H}$-synchronizing coloring. The value of $k$ is equal to the sum of all such $k_{H}$ (Theorem 3). The search of $k$ is linear.

The edges outside subgraphs $H$ can be colored arbitrarily (sometimes by additional colors). The outgoing edges of every state are colored by different colors.

\section{The algorithm for $k$-synchronizing coloring}

Let us begin from an arbitrary coloring of a directed graph $\Gamma$ with $n$ vertices and $d$ outgoing edges of any vertex. The considered $d$ colors define $d$ spanning subgraphs of the graph. We find all $S C C H$ having no outgoing edges and study every such $S C C H$ separately.

We keep images of vertices and colored edges from the generic graph by any transformation and homomorphism.

If there exists a loop in $\Gamma$ then let us color the edges of a tree with root in the vertex of a loop by one color. The other edges may be colored arbitrarily. The coloring in this case is synchronizing for the considered $S C C$.

In the case of two incoming bunches of one vertex the beginnings of these bunches form a stable pair by any coloring (Lemma 4). We merge both vertices in the homomorphic image of the graph (Theorem 1) and obtain according to the theorem a new graph of the size less than $|\Gamma|$.

The linear search of two incoming bunches and of loop can be made at any stage of the algorithm.

Find the parameters of the spanning subgraph: levels of all vertices, the number of vertices (edges) in cycles, for any vertex let us keep its tree and the cycle of the root of the tree. We form the set of vertices of maximal level and choose from the set of trees a tree $T$ with vertex $\mathbf{p}$ of maximal level. This step is linear and used by any recoloring.

Lemma 10. Let graph $\Gamma$ have two cycles $C_{u}$ and $C_{v}$ either with one common vertex $\mathbf{p}_{1}$ or with a common sequence $\mathbf{p}_{1}, \ldots, \mathbf{p}_{k}$, such that all incoming edges of $\mathbf{p}_{i}$ form a bunch from $\mathbf{p}_{i+1}(i<k)$. Let $u \in C_{u}$ and $v \in C_{v}$ be the edges of the cycles leaving $\mathbf{p}_{1}$. Let $T$ be a maximal subtree of $\Gamma$ with the root $\mathbf{p}_{1}$ and edges from $C_{u}$ and $C_{v}$ except $u$ and $v$.

Then the the adding one of the edges $u$ or $v$ turns the subtree $T$ into precisely one maximal tree of the spanning subgraph.

Proof. Let us add to $T$ either $u$ or $v$ and then find the maximal levels of vertices in both cases. The vertex $\mathbf{p}_{i}$ for $i>1$ could not be the root of a tree. If any tree of spanning subgraph with vertex of maximal level has the root $\mathbf{p}_{1}$ then in both cases the lemma holds. If some tree of spanning subgraph with vertex of maximal level has the root only on $C_{u}$ then let us choose the adding of $v$. In this case the level of the considered vertex is growing and only the new tree with 
root $\mathbf{p}_{1}$ has vertices of maximal level. In the case of the root on $C_{v}$ let us add $u$.

1) If there are two cycles with one common vertex then we use Lemma 10 and find a spanning subgraph $R_{1}$ such that any vertex $\mathbf{p}$ of maximal level $L$ belongs to one tree with root on a cycle $H$. Then after coloring edges of $R_{1}$ with the color $\alpha$ we find stable pair $\mathbf{q}=\mathbf{p} \alpha^{L-1+|H|}$ and $\mathbf{s}=\mathbf{p} \alpha^{\mathbf{L}-\mathbf{1}}$ (Lemma 7) and go to step 3). The search of a stable pair in this case is linear and the whole algorithm therefore is quadratic.

2) Let us consider now the three replacements from Lemma 7 and find the number of edges in cycles and other parameters of the spanning subgraph of the given color. If the number of edges in the cycles is growing, then the new spanning subgraph must be considered and the new parameters of the subgraph must be found. In the opposite case, after at most $3 d$ steps, by Lemma 7 , there exists a tree $T_{1}$ with root on the cycle $H_{1}$ of a spanning subgraph $R_{1}$ such that any vertex $\mathbf{p}$ of maximal level $L$ belongs to $T_{1}$.

Suppose the edges of $R_{1}$ are colored by the color $\alpha$. Then the vertices $\mathbf{q}=$ $\mathbf{p} \alpha^{L-1+\left|H_{1}\right|}$ and $\mathbf{s}=\mathbf{p} \alpha^{\mathbf{L - 1}}$ by Lemma 7 form a stable pair.

3) Let us finish the coloring and find the subsequent stable pairs of the pair $(\mathbf{s}, \mathbf{q})$ using appropriate coloring. Then we go to the homomorphic image $\Gamma_{i} / \rho$ (Theorem 1) of the considered graph $\Gamma_{i}\left(O\left(\left|\Gamma_{i}\right| m_{i} d\right)\right.$ complexity where $m_{i}$ is the size of the map $\Gamma_{i}$ ). Then we repeat the procedure with a new graph $\Gamma_{i+1}$ of a smaller size. So the overall complexity of this step of the algorithm is $O\left(n^{2} d\right)$ in the majority of cases and $O\left(n^{3} d\right)$ if the number of edges in cycles grows slowly, $m_{i}$ decreases also slowly, loops do not appear and the case of two ingoing bunches rarely emerges (the worst case).

Let $\Gamma_{i+1}=\Gamma_{i} / \rho_{i+1}$ on some stage $i+1$ have $k$-synchronizing coloring. For every stable pair $\mathbf{q}, \mathbf{p}$ of vertices from $\Gamma_{i}$ there exists a pair of corresponding outgoing edges that reach either another stable pair or one vertex. This pair of edges is mapped on one image edge of $\Gamma_{i+1}$. So let us give the color of the image to preimages and obtain in this way a $k$-synchronizing coloring of $\Gamma_{i}$. This step is linear. So the overall complexity of the algorithm is $O\left(n^{3} d\right)$ in the worst case. If Lemma 10 is applicable, then the complexity is reduced at this branch of the algorithm (as well as at some other branches) to $O\left(n^{2} d\right)$. The practical study of the implementation of the algorithm demonstrates mainly $O\left(n^{2} d\right)$ time complexity.

\section{Conclusion}

In this paper, we have continued the study of $k$-synchronizing coloring of a finite directed graph. It is an important and natural generalization of the well known notion of the synchronizing coloring. This new approach gives an opportunity to extend essentially the class of studied graphs, removing the restriction on the size of the great common divisor of the lengths of the cycles of the graph. The restriction is inspired by the Road Coloring Problem and now for $k$-synchronizing coloring can be omitted. 
There exists still another restriction on the properties of the considered graphs concerning the uniform outdegree of the vertex. However, it also can be omitted by consideration of subgraphs of the graph (Corollary 8).

The practical use of this investigation needs an algorithm and the paper presents a corresponding polynomial time algorithm of the road coloring for the $k$-synchronization implemented in the package TESTAS. The realization of the algorithm demonstrates a new linear visualization program [3].

\section{References}

1. R.L. Adler, and B. Weiss, Similarity of automorphisms of the torus, Memoirs of the Amer. Math. Soc., Providence, RI, 98(1970).

2. A. Aho, J. Hopcroft, and J. Ulman. The Design and Analisys of Computer Algorithms, Addison-Wesley 1974.

3. T. Bauer, N. Cohen, and A.N. Trahtman, The visualization of digraph based on its structure properties. RTAGS, Ramat-Gan, Israel, 2007.

4. M.P. Béal, and D. Perrin, A quadratic algorithm for road coloring, arXiv: 0803.0726v2 [cs.DM], 2008.

5. G. Budzban, and P. Feinsilver, The Generalized Road Coloring Problem. arXiv:0903.0192v, [cs.DM], 2008.

6. K. Culik, J. Karhumaki, and J. Kari, A note on synchronized automata and Road Coloring Problem, Developments in Language Theory (5th Int. Conf., Vienna, 2001), Lecture Notes in Computer Science, 2295(2002), 175-185.

7. J. Černy, Poznamka k homogenym eksperimentom s konechnymi automatami, Math.-Fyz. Čas., 14, 1964, 208-215.

8. J. Kari, Synchronizing finite automata on Eulerian digraphs, Springer, Lect. Notes in Comp. Sci., 2136(2001), 432-438.

9. A. Mateescu, and A. Salomaa, Many-valued truth function. Černy conjuncture and road coloring, EATCS Bulletin, 68134-150, 1999.

10. A.N. Trahtman, Synchronizing Road Coloring. 5-th IFIP WCC-TCS, Springer, 273(2008), 43-53.

11. A.N. Trahtman, The Road Coloring and Cerny Conjecture. Proc. of Prague Stringology Conference, 2008, 1-12.

12. A.N. Trahtman, The Road Coloring for Mapping on k States. arXiv:0812.4798, 2008 [cs.DM].

13. A.N. Trahtman, The road coloring problem. Israel Journal of Math., v.1, 172(2009), 51-60. 